UMD-PP-10-013

\title{
Direct Gaugino Mediation
}

\author{
Daniel Green,` Andrey Katz,* and Zohar Komargodski \\ - School of Natural Sciences, Institute for Advanced Study, Princeton, NJ 08540 \\ * Department of Physics, University of Maryland, College Park, MD 20742
}

\begin{abstract}
We describe renormalizable supersymmetric four-dimensional theories which lead to gaugino mediation and various generalizations thereof. Even though these models are strongly coupled, we can demonstrate the parametric suppression of soft scalar masses via Seiberg duality. For instance, we show that our models have a parameter which continuously interpolates between suppressed soft scalar masses and their conventional gauge mediated contribution. The main physical effect which we utilize is the general relation between massive deformations in one frame and higgsing in the dual frame. Some compelling and relatively unexplored particle physics scenarios arise naturally in this framework. We offer preliminary comments on various aspects of the phenomenology and outline several of the outstanding open problems.
\end{abstract}

August/2010 


\section{Introduction}

Weak scale supersymmetry (SUSY) is an attractive framework for addressing the hierarchy problem. Within the context of the Minimally Supersymmetric Standard Model (MSSM), the Higgs sector can be made natural provided the soft masses for the gauginos and stops are both comparable to the weak scale. Achieving such a spectrum while solving the flavor problem has long been a benchmark for successful model building. Gauge mediation [1] 8 ] is a promising scenario as it solves the flavor problem by construction. Furthermore, the simplest schemes for gauge mediation, like minimal gauge mediation [6-8], also provide a desirable spectrum of soft masses.

Since the work of ISS [9], there has been a rejuvenated interest in embedding gauge mediation in models of dynamical SUSY breaking (for many relevant references see the recent review [10]). However, in most cases the sparticles turned out to be heavy compared to the gauginos. This is associated to the need to break $R$-symmetry in order to generate gaugino masses, and in addition, to constraints on the vacuum structure of the theory [11,12].

Gaugino mediation [13,14] is a different scheme of mediation that gives a spectrum where the scalar masses are no larger than the gaugino masses. Gaugino mediation arises when there is some mechanism that screens the scalar masses from SUSY breaking without altering the gaugino masses. As a result, the scalar masses are smaller than the gaugino masses at high energies but are generated by renormalization group flow in the MSSM. There are several proposals for how to achieve such screening, starting with no-scale supergravity [15, 16] and including large extra dimensions [13, 14], deconstruction [17-20], large anomalous dimensions [21,22], or large numbers of messengers. However, some of these scenarios are not on theoretically firm grounds; for example, some run into strong coupling and are, strictly speaking, incalculable, while others lack consistent examples or UV completions.

In this paper we construct renormalizable four-dimensional dynamical models of SUSY breaking that lead to scalar screening. We will also show that such models are generic. The existence of a calculable framework allows detailed phenomenological studies, and indeed, models of scalar screening can lead to novel, well-motivated, particle physics scenarios. At low energies, the effective Lagrangians of our models describe a sequence of symmetry breaking phenomena. The structure of the effective theory has some features in common 
with the ansatz arising via deconstructing higher dimensional models [17 20]. The suppression of the scalar masses is given either by the ratio of some higgsing scale $v$ and the messenger mass $M$, or additional loop factors.

Of course, our results differ from simple deconstructed models. Their ad-hoc structure of higgsing and "link fields" arises in our case dynamically from a single sector theory, which undergoes strong dynamics along its flow. The relation between models inspired by the ansatz of deconstruction and our dynamical models is analogous to the relation between O'Raifeartaigh-like models and calculable models of dynamical SUSY breaking: calculable models of dynamical SUSY breaking often reduce to O'R-like models in the IR. Indeed, similarly to O'R-like models, deconstructed models leave many conceptual questions open. Finding elegant dynamical models which flow to them provides some answers. On the other hand, this analogy also suggests of the universality of deconstructed models and their importance, something we believe in strongly.

Even though we discuss strongly coupled theories, we are able to analyze them thanks to Seiberg duality [23]. A well known phenomenon in electric-magnetic duality is that a mass term in one duality frame is described by higgsing in the other duality frame. As we will explain, certain patterns of higgsing are sufficient for generating suppression factors in the soft scalar masses. This suggests that gaugino mediation may arise from mass deformations of asymptotically free theories with IR free magnetic dual descriptions. Luckily, the same kind of deformations lead to metastable SUSY breaking [9] and so we claim that theories with suppressed scalar masses are generic in this class.

Strictly speaking, our models fall into the class of General Gauge Mediation (GGM) as defined in [24], so they are really dynamical models of gauge mediation. However, they can yield spectra far from those we are used to. We have therefore chosen to refer to them as "Direct Gaugino Mediation" models. 1

The plan for the paper is as follows. In section 2, we explain the low energy theory of scalar screening in detail, including how to understand it in the context of general gauge mediation. We will see that the standard model charges of the messengers are effectively functions of energy. In section 3, we will present detailed models based on deformations of SQCD with $S U(N)$, and further generalize to $S O(N)$ and $S p(N)$ gauge groups. In section 4 , we will include more phenomenologically relevant details of the model and many

1 The analysis of 24 has stimulated various attempts to generalize the minimal setup of gauge mediation. A partial list of such works is 25.34. 
model building applications. We will speculate about the kind of spectra such models can lead to, and discuss preliminary aspects of the phenomenology. Section 5 contains a summary and an outlook.

\section{From Gauge to Gaugino Mediation}

We begin this section with a review of some known facts about gauge mediation and introduce the question of gaugino mediation. This is also our opportunity to explain in more detail what we are after in this paper. On occasion, we use the language of GGM to shed some further light on the problem.

Gauge mediation is a compelling way of mediating SUSY breaking in some hidden sector $H$ to the MSSM. We assume that the flavor symmetry of $H, F_{H}$, contains the MSSM group $G_{M S S M} \subset F_{H}$ and we gauge this subgroup. If the typical mass of particles in $H$ is $M$ and the SUSY breaking scale is $F$ then we expect that this mechanism generates soft scalar and gaugino masses of the form

$$
m_{\widetilde{f}}^{2} \sim \frac{\alpha^{2}}{(4 \pi)^{2}} \frac{F^{2}}{M^{2}}, \quad m_{\tilde{g}} \sim \frac{\alpha}{4 \pi} \frac{F}{M} .
$$

This has all the usual nice features of gauge mediation, especially that it is flavor blind and that the gaugino and scalar soft masses are comparable.

We can test (2.1) in many of the calculable models of SUSY breaking. It is rather easy to engineer models with messengers which lead to (2.1), but the success with dynamical models has been limited. In many of these models the scalar masses are too large compared to the gaugino masses (unless cumbersome tricks are employed, e.g. 355 38]) and so the resulting spectrum is fine tuned.

In this paper we would like to look for models which lead dynamically to boundary conditions with $m_{\tilde{g}} \gg m_{\widetilde{f}}$. In light of the description of the situation with gauge mediation above, this goal might seem hopeless, but we will see that in fact the opposite is true. Let us describe an ansatz for how this can be achieved in principle [19,20].

Consider a copy of the SM group $G_{S M}^{(2)}$ embedded in the flavor group $F_{H}$ of some SUSY-breaking sector (with typical scale $M$ and SUSY breaking scale $F$ ). Then we have some sector of "link fields" which are charged under both $G_{S M}^{(2)}$ and another copy of the SM group, $G_{S M}^{(1)}$. The sector of link fields is assumed to be supersymmetric when we turn 
off the gauge coupling of $G_{S M}^{(2)}$. An important dynamical assumption is that the sector of link fields breaks the symmetry

$$
G_{S M}^{(1)} \times G_{S M}^{(2)} \hookrightarrow G_{S M}
$$

at some scale $v$. This symmetry breaking should take place in the supersymmetric limit, when the gauge coupling of $G_{S M}^{(2)}$ is zero. The observable gauge fields are identified with the unbroken ones, $G_{S M}$. All the MSSM matter fields are assumed to be charged only under $G_{S M}^{(1)}$. The situation is summarized in Fig.1.

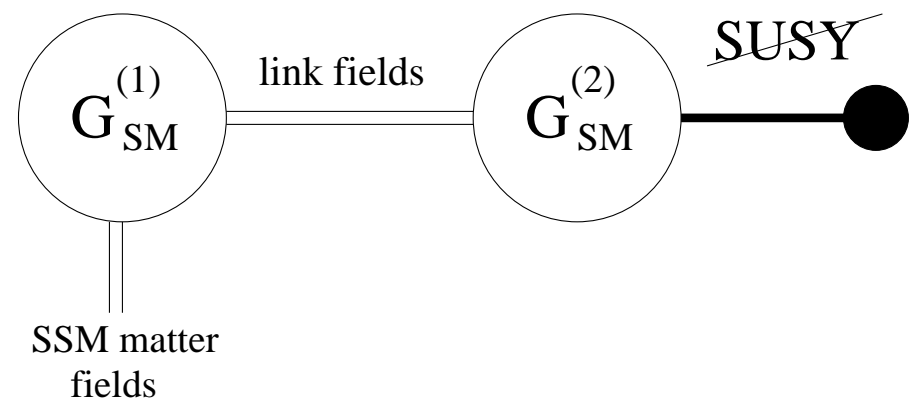

Figure 1: A schematic picture of the deconstruction ansatz for gaugino mediation.

To understand what these models do, we first assume that $v \gg M$. Below the scale $v$ the theory is still supersymmetric, including the hidden sector. Both the hidden sector and the visible sector are charged under $G_{S M}$. Thus, it looks precisely like the usual setup of gauge mediation with $G_{S M}$ being embedded in the flavor group $F_{H}$. We recover the conventional paradigm and predictions. Now let us assume that $v \ll M$. This limit is much more interesting. Here, at energies above $v$ the visible matter fields do not interact with the SUSY breaking sector up to diagrams with four loops in the various gauge couplings. So the contribution from these energy scales is negligible and can be estimated as $m_{\widetilde{f}}^{2} \sim \frac{\alpha^{4}}{(4 \pi)^{4}} \frac{F^{2}}{M^{2}}$. At energies below $v$ the group breaks (2.2) and now there are interactions at two loops and three loops between the sparticles of the MSSM and the SUSY breaking sector. Since $v \ll M$ these two loop interactions cannot probe energy scales of order $M$ and the scalar masses come out suppressed $m_{\widetilde{f}}^{2} \sim \frac{\alpha^{2}}{(4 \pi)^{2}} \frac{F^{2}}{M^{2}} \frac{v^{2}}{M^{2}}$. Note the $v^{2} / M^{2}$ suppression compared to (2.1). Some three loop interactions are important as well, but we will postpone this topic to section 4 .

To summarize, these models with $v \ll M$ give rise to scalar masses which are parametrically smaller than the conventional estimate. Their actual value at the boundary is 
determined by whether the $v^{2} / M^{2}$ suppression is larger or smaller than the additional loop factor suppression.

The gaugino soft mass behaves very differently (and much simpler). As far as the the gauge group $G_{S M}^{(2)}$ is concerned, we generate, via the usual gauge mediation mechanism, gaugino mass of the form $m_{\widetilde{g}^{(2)}} \sim \frac{\alpha}{4 \pi} \frac{F}{M}$. (Of course in this case, by $\alpha$ we mean $\alpha_{2}$, but we prefer not to clutter the notation at this stage.) The breaking to the diagonal implies that the actual visible gaugino is a linear combination of $\widetilde{g}^{(1)}$ and $\widetilde{g}^{(2)}$. This means that it directly acquires the soft mass of $\widetilde{g}^{(2)}$. This soft mass is, consequently, independent of the higgsing scale $v$. In other words, the soft mass of the physical low energy gaugino is $m_{g} \sim \frac{\alpha}{4 \pi} \frac{F}{M}$.

We see that the ansatz of Fig.1 with $v \ll M$ gives rise to boundary conditions in which the gauginos are parametrically heavier than the sparticles.

For an alternate explanation of why the gaugino is unsuppressed (and independent of $v$ ) while the scalars are, it is useful to invoke the language of GGM. If we think about the link fields together with the SUSY breaking sector $H$ and the gauge fields $G_{S M}^{(2)}$ as one hidden sector, then this is the setup of GGM. We can write the scalar masses in terms of correlation functions of $G_{S M}^{(1)}$ global currents as?

$$
m_{\widetilde{f}}^{2} \sim g_{1}^{4} \int \frac{d^{4} p}{p^{2}}\left(C_{0}^{(1)}\left(p^{2}\right)-4 C_{1 / 2}^{(1)}\left(p^{2}\right)+3 C_{1}^{(1)}\left(p^{2}\right)\right)
$$

The superscript is to remind that these are correlation functions associated to the global symmetry $G_{S M}^{(1)}$. The gaugino mass is given by

$$
m_{\tilde{g}} \sim g_{1}^{2} B_{1 / 2}(p=0)
$$

The key difference between the scalar and gaugino mass is that the former is sensitive to correlation functions at all momenta scale while the latter is a zero momentum observable. In our specific ansatz for the hidden sector, the SUSY breaking fields are really decoupled from the current multiplets of $G_{S M}^{(1)}$ for $p \gg v$. This effectively means that the integral (2.3) is cutoff at $p \sim v$. This in turn is responsible for a parametric suppression of the scalar masses if $v \ll M .3$ On the other hand, the gaugino mass is given by a zero

2 We are using the notation of 24].

3 A corollary from this description is that the leading order in $v^{2} / M^{2}$ depends only on the zero-momentum value of $C_{0}-4 C_{1 / 2}+3 C_{1}$. 
momentum correlation function, and thus it is oblivious to all the complicated symmetry breaking patterns at nonzero energy.

Physically we can think of these models as giving the messenger fields charge under the MSSM only at energy scales below $v$. In other words, we effectively assign charges which are energy dependent. This does not affect the gauginos but creates a suppression for the scalars (under the conditions we discussed). In section 4 we mention some of the many possible phenomenological consequences.

It is important to keep in mind that the MSSM RG flow gives rise to a contribution to the soft scalar masses of the following form

$$
\delta m_{\widetilde{f}}^{2} \sim \frac{\alpha}{4 \pi} m_{\tilde{g}}^{2}
$$

This can be interpreted as a contribution to the soft scalar masses at three loops and it is further logarithmically enhanced if the mediation scale is high. Hence, unless some tuning is invoked, we do not expect to be able to make the gauginos of the MSSM heavier than the scalars by more than a factor of a few. The details depend on the species of particles we are interested in. Thus, the practically interesting values of $v / M$ cannot be much smaller than $\mathcal{O}\left(10^{-1}\right)$.

While the ansatz of Fig.1 is interesting, it seems baroque. The suppression of the scalar masses is highly dependent on the fact that the link fields are supersymmetric for vanishing $G_{S M}^{(2)}$ gauge coupling. Further, no fields in the SUSY-breaking sector can be charged under $G_{S M}^{(1)}$. To get the suppression we also need to require the higgsing scale $v$ to be smaller than the messenger scale $M$. Since eventually we need to look for dynamical models, preferably as simple as possible, the natural question is whether the involved structure of Fig.1 can emerge from the dynamics of some "single sector" model. This may seem unlikely because in such models all the particles interact strongly with all the others. Then, we expect that all the fields feel SUSY breaking equally.

This intuition is indeed correct in many cases, and the scalar suppression is washed out. But there is a large class of single-sector theories which can be analyzed and shown to lead to spectra with $m_{\widetilde{f}} \ll m_{\tilde{g}}$. We give such examples in the next section and explain why the mechanism we present is generic. 


\section{Dynamical Models}

The main conceptual ingredient we will utilize is that higgsing in the magnetic low energy effective theory corresponds to adding a mass term in the electric theory. As we saw in the previous section, higgsing is an important ingredient in realizing this sfermion mass screening mechanism. In addition, adding mass terms in the UV gives rise generically to metastable SUSY breaking vacua. This is why, in fact, many dynamical models can lead in the IR to both SUSY breaking and suppressed scalar masses.

Of course, we have to look for models with an appropriate sector of link fields. As we explained, our increased understanding of these strongly coupled theories has shown that such features, even though they may not seem intuitive, can arise generically. This does not mean that they always occur. Indeed, in the simplest version of massive SQCD, it seems impossible to find a mechanism to suppress the scalar masses. see next, massive SQCD with vector like Yukawa couplings has an effective theory which drives spontaneous SUSY breaking, on top of the structure we presented in section 2.

\subsection{Massive $S Q C D$ with Yukawa Interactions}

Consider $S U\left(N_{c}\right)$ SQCD with $N_{f}$ quarks $Q^{I}$ in the fundamental representation and $N_{f}$ quarks $\widetilde{Q}_{I}$ in the anti-fundamental representation. We divide them into two subsets of $k$ and $N_{f}-k$ quarks, labeled with the indices $i=1 \ldots k$ and $a=k+1 \ldots N_{f}$. We also introduce some singlets $S_{i}^{a}$ and $\widetilde{S}_{a}^{i}$. The theory is simply given by

$$
W_{\text {electric }}=m_{I}^{J} Q^{I} \widetilde{Q}_{J}+S_{i}^{a} Q^{i} \widetilde{Q}_{a}+\widetilde{S}_{a}^{i} Q^{a} \widetilde{Q}_{i}
$$

To preserve a large flavor symmetry group we choose the mass matrix to be $m_{I}^{J}=m_{1} \delta_{i}^{j} \oplus$ $m_{2} \delta_{a}^{b}$, so quarks in the first set have mass $m_{1}$ and in the second set $m_{2}$. If we also include a mass term $m_{S} S_{a}^{i} \widetilde{S}_{i}^{a}$, then this theory is the most general renormalizable theory respecting the $S U(k) \times S U\left(N_{f}-k\right)$ flavor group. For now we treat this group as global (although later we will gauge parts of it).

4 The main trouble is the fact that we typically get messengers charged under the SUSYbreaking flavor group directly. In other words, there is no good sub-sector of link fields.

5 Some aspects of SQCD with singlets coupled to the quarks were analyzed in [39]. Possible applications of this theory were discussed, for example, in 40-43. However, in our case the singlets are not strictly necessary; the Yukawa couplings can be replaced by dangerously irrelevant operators composed out of electric quarks. 
The theory also has two abelian symmetries. One is the usual baryon number $U(1)_{B}$. The second assigns charge \pm 1 to $Q^{i}, \widetilde{Q}_{i}$ respectively and \pm 1 to $\widetilde{S}_{a}^{i}, S_{i}^{a}$. We denote this symmetry by $U(1)^{\prime}$. These symmetries are not anomalous. They also have no cubic anomalies.

When all the mass terms are set to zero the theory has a new global symmetry. It is therefore natural to imagine these masses are small (compared to the strong coupling scale). In this regime the mass terms for the quarks are physically crucial, but one can set $m_{S}=0$ without changing any of the essential details of the analysis. This is why we do not include the mass term explicitly in (3.1) and do not discuss it any further.

For $N_{f}<3 N_{c}$ the gauge coupling grows strong in the IR and all the matter fields interact strongly with each other. In spite of that, we will show (for some regime of parameters) that this theory in fact reduces precisely to the ansatz of the previous section. We will find that the scales $m_{1}, m_{2}$ are related to $v, M$.

We concentrate on $N_{f}<3 N_{c} / 2$, where at low energies and small VEVs, the theory flows to the IR-free Seiberg dual description.6 The superpotential is

$$
W_{\text {magnetic }}=q M \widetilde{q}+\Lambda S_{i}^{a} M_{a}^{i}+\Lambda \widetilde{S}_{a}^{i} M_{i}^{a}+\Lambda \operatorname{Tr}(m M) .
$$

The magnetic gauge group is $S U\left(N_{f}-N_{c}\right)$. The magnetic quarks $q, \widetilde{q}$ are charged, while the mesons $M$ and the original singlets $S, \widetilde{S}$ are neutral.

We take $m_{1,2} \ll \Lambda$ and denote $\mu_{1,2}^{2}=-m_{1,2} \Lambda$. The theory (3.2) is weakly coupled. We see that the mesons $M_{i}^{a}, M_{a}^{i}$ are massive with mass of order $\Lambda$ and can be integrated out. To write the resulting action we parametrize the $N_{f}$ magnetic quarks as $q=\left(\chi_{i}, \psi_{a}\right)$, $\widetilde{q}=\left(\widetilde{\chi}^{i}, \widetilde{\psi}^{a}\right)$. After integrating out the heavy states, the theory at low energies is

$$
W=\chi N \widetilde{\chi}+\psi M \widetilde{\psi}-\mu_{1}^{2} N_{i}^{i}-\mu_{2}^{2} M_{a}^{a} .
$$

In the above we have decomposed the meson such that the upper block is denoted $N$ and the lower one $M$

$$
M_{N_{f} \times N_{f}}=\left(\begin{array}{cc}
N_{k \times k} & 0 \\
0 & M_{\left(N_{f}-k\right) \times\left(N_{f}-k\right)}
\end{array}\right) \text {. }
$$

The off-diagonal components were integrated out using the equations of motion of the $S, \widetilde{S}$ superfields. Therefore, the low energy effective theory consists of two sectors which interact

6 We are careless about the distinction between the electric and magnetic strong scales. For simplicity, we assume that they are identical and drop all the incalculable numbers in the dual description. 
arbitrarily weakly at the IR, through the magnetic gauge fields. This is an emergent phenomenon.

The analysis of both of these sectors is straightforward. To make the discussion even simpler we choose $k=N_{f}-N_{c} \equiv N$, although analogous results hold as long as either $k \leq N$ or $k \geq N_{c}$.

Let us start from analyzing the sector of the $\psi$ quarks. There are $N_{f}-N=N_{c}$ flavors of $\psi$ quarks. Since in the free magnetic phase $N_{c}>N$, we cannot satisfy the $F$-term equations for the $M_{a}^{b}$ mesons and SUSY is broken for small field VEVs. Note the similarity of this sector to the ISS model [9], except that the number of quarks is $N_{c}$ rather than $N_{f}$. We will see that although this sector is indeed similar, other aspects of the model are qualitatively different.

It is convenient to further decompose the quarks and mesons as follows

$M=\left(\begin{array}{cc}X_{N_{f}-N_{c}} & Y \\ \widetilde{Y} & Z_{2 N_{c}-N_{f}}\end{array}\right), \quad \psi=\left(\lambda_{N_{f}-N_{c}}, \rho_{2 N_{c}-N_{f}}\right), \quad \widetilde{\psi}=\left(\widetilde{\lambda}_{N_{f}-N_{c}}, \widetilde{\rho}_{2 N_{c}-N_{f}}\right)$.

In the attempt to cancel as many $F$-terms as possible we expand around $X=0, \lambda=\widetilde{\lambda}=$ $\mu_{2}$ II. This breaks the symmetries as follows

$$
S U(N)_{\chi} \times S U\left(N_{c}\right)_{\psi} \times[S U(N)] \hookrightarrow S U(N)_{\chi} \times S U\left(2 N_{c}-N_{f}\right)_{\rho} \times S U(N)_{\text {diagonal }}
$$

We have added subscripts to the various symmetry groups to make it obvious how they act. $S U(N)_{\text {diagonal }}$ is a diagonal combination of the magnetic group and the $S U(N)_{\lambda}$ flavor

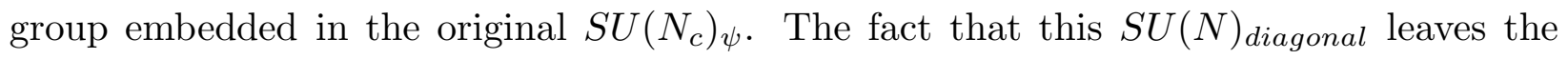
vacuum invariant and mixes with the magnetic group will have far reaching consequences. This is the seed for the higgsing phenomenon we need to realize the scenario outlined in section 2. Note that the magnetic gauge group is emergent, so this seed is not even visible in the original description of the theory.

To analyze the spectrum of this vacuum we first turn off the magnetic gauge group and treat it as a global symmetry. First of all, we find all the Nambu-Goldstone bosons associated to this breaking. There are also pseudo-moduli $\operatorname{Re}(\lambda-\widetilde{\lambda})$ and $Z$. The modes in $\operatorname{Re}(\lambda-\widetilde{\lambda})$ obtain positive mass squared at one-loop and are thus set to the origin. Similarly, a one-loop Coleman Weinberg potential sets $Z=0$ as well. Therefore, this is a good SUSY-breaking vacuum, with no noncompact massless fields. Reintroducing the magnetic gauge coupling, we find that some of the Goldstone bosons are eaten and that 
some of the pseudo-moduli obtain an additional positive mass-squared contribution. The vacuum above is obviously $D$-flat.

We now turn to analyzing the sector of the $\chi$ quarks. The rank of the matrix $\chi \widetilde{\chi}$ is at most $N_{f}-N_{c}$, and this can be arranged to cancel all the $F$-terms of $N_{i}^{j}$,

$$
\langle\chi \tilde{\chi}\rangle=\mathbb{1}_{N} \mu_{1}^{2}
$$

We can use the unbroken $S U(N)_{\chi} \times S U(N)_{\text {diagonal }}$ to diagonalize $\chi, \chi=\mu_{1} \operatorname{diag}\left(a_{i}\right)$, which guarantees by (3.7) that $\tilde{\chi}$ is diagonal as well $\tilde{\chi}=\mu_{1} \operatorname{diag}\left(a_{i}^{-1}\right)$. Further, the energy is minimized as long as the $D$-terms of the magnetic group vanish. This is achieved if $a_{i}=a$ for some general complex $a$. Th The last remaining noncompact modulus is fixed to $a=1$ if we gauge the vector like $U(1)^{\prime}$ symmetry. Thus we summarize that our vacuum is

$$
\langle\chi\rangle=\mu_{1} \mathbb{I}_{N}, \quad\langle\widetilde{\chi}\rangle=\mu_{1} \mathbb{I}_{N}
$$

This vacuum further breaks the symmetry (3.6)

$$
S U(N)_{\chi} \times S U\left(2 N_{c}-N_{f}\right)_{\rho} \times S U(N)_{\text {diagonal }} \hookrightarrow S U(N)_{\text {visible }} \times S U\left(2 N_{c}-N_{f}\right)_{\rho}
$$

Note that this last step contains another crucial ingredient for achieving the mechanism of section 2 ; the breaking pattern via the $\chi$ quarks (which are supersymmetric at tree-level) is of the form $S U(N) \times S U(N) \hookrightarrow S U(N)$.

Therefore, somewhat surprisingly, the simple theory (3.1) has a very rich structure in the IR, accommodating all the necessary ingredients for suppressed scalar masses. This theory, in spite of all its components being strongly interacting at some intermediate scale, reduces in the IR to two weakly interacting sectors, one breaks SUSY and the other does not. These sectors communicate via the magnetic gauge fields. The mass terms in the UV trigger higgsing of the magnetic group. This crucial feature is a general phenomenon in theories with electric-magnetic duality. The flavor symmetries mix with the magnetic group generators and eventually break further to diagonal combinations.

7 We are using the magnetic $D$-terms to conclude that $a_{i}=a$ for all $i$, but one could worry that it is possible to involve the $\chi$ quarks too and find more complicated vacua. This does not happen because of the one-loop positive masses the $\chi$ quarks obtain, they are fixed to the VEV we discussed after (3.5). 
The dictionary between this model and section 2 is straightforward. We can weakly gauge $8 U(N)_{\chi} \times S U(N)_{\lambda}$ and let the matter fields of the MSSM carry just $S U(N)_{\chi}$ charges (of course the simplest choice is to take $N=5$ ). The SUSY breaking scale (which is roughly also the messenger mass in this model) is $\mu_{2}$ while the higgsing scale is $\mu_{1}$. The link fields are the $\chi$ magnetic quarks. Hence, the scalar mass squared will be suppressed roughly by $\mu_{1}^{2} / \mu_{2}^{2}$ relative to the naive value. For convenience, we summarize the dictionary between the deconstruction ansatz and the dynamical model in the following table:

\begin{tabular}{|c|c|}
\hline Deconstruction & Dynamical Model \\
\hline gauge group $G_{S M}^{(1)}$ & $S U(N)_{\chi}$ \\
\hline gauge group $G_{S M}^{(2)}$ & $S U(N)_{\lambda}$ \\
\hline SUSY breaking scale $M$ & $\sqrt{m_{2} \Lambda}$ \\
\hline higgsing scale $v$ & $\sqrt{m_{1} \Lambda}$ \\
\hline link fields & $\chi, \tilde{\chi}$ \\
\hline
\end{tabular}

Recall that, in the magnetic duality frame, the global symmetry $S U(N)_{\lambda}$ mixes with the magnetic gauge bosons to become $S U(N)$ diagonal, which in the end of the day plays the role of $G_{S M}^{(2)}$.

The ratio of $\mu_{1}^{2} / \mu_{2}^{2}$ cannot be made arbitrarily small. The main obstruction (common to all deconstruction-like theories) is that the $\chi$ quarks receive some soft masses through the magnetic gauge fields. These should not compete with the dynamics leading to spontaneous symmetry breaking, so $\mu_{1}^{2} / \mu_{2}^{2}>10^{-4}$. As we have explained in section 2 , this constraint is irrelevant from a practical point of view and can still accommodate any conceivable scalar suppression.

The SUSY-breaking vacuum we found here is metastable. Supersymmetric vacua are restored via non-perturbative effects which we discuss in the next subsection.

The construction we presented here is strongly dependent on the mixing of flavor symmetries with the magnetic gauge group. (This should perhaps be called "magnetic color flavor locking." ) Since this is a general property of massive theories with a dual description,

8 Before gauging any flavor symmetries, there are degenerate vacua where $\lambda=\widetilde{\lambda}=0$ and $\widetilde{\rho}_{i}=\rho_{i} \neq 0$. These vacua do not give the desired pattern of higgsing. Even though, strictly speaking, for our vacuum to exist this degeneracy does not have to be lifted, it is automatically removed by several mechanisms which are described in section 4 . 
our model is in fact easily generalizable to many other examples. In subsection 3.3 we briefly discuss some analogous $S O(N) / S p(N)$ theories.

As it stands, the theory at hand has an accidental unbroken $R$-symmetry in the IR and so this scalar suppression is not of much use phenomenologically. This is a minor technical issue and there are many ways around it. We list some of the simplest ones in section 4 , where we also make some rough estimates of the soft scalar and gaugino masses in this theory.

\subsection{Non-Perturbative Restoration of Supersymmetry}

The search for SUSY vacua can be carried either in the electric or in the magnetic description, with identical results. Here we will only discuss the electric description. Furthermore, since SUSY vacua are protected by holomorphy we are allowed to study (3.1) with masses $m \gg \Lambda$ and then analytically continue the results to the regime of interest. (We again set the mass term for the singlets to zero for simplicity.) This theory is conceptually easy to understand. Below the scale $\sim m$ it flows to pure SYM theory and singlets. The scale of this pure SYM theory depends on the singlets as

$$
\Lambda_{e f f}^{3 N_{c}}=\operatorname{det} \mathcal{M} \Lambda^{3 N_{c}-N_{f}}
$$

where

$$
\mathcal{M}=\left(\begin{array}{cc}
m_{1} \mathbb{I}_{k} & S \\
\widetilde{S} & m_{2} \mathbb{I}_{N_{f}-k}
\end{array}\right) .
$$

This creates a superpotential due to gaugino condensation

$$
W_{e f f} \sim(\operatorname{det} \mathcal{M})^{\frac{1}{N_{c}}} \Lambda^{3-\frac{N_{f}}{N_{c}}}
$$

The VEVs of the mesons depend on $S$ and are given by

$$
\langle Q \widetilde{Q}\rangle \sim\left(\operatorname{det} \mathcal{M} \Lambda^{3 N_{c}-N_{f}}\right)^{\frac{1}{N_{c}}} \mathcal{M}^{-1}
$$

As mentioned before, some of the interesting values of $k$ are $k \leq N_{f}-N_{c}$. One simple property of the determinant of (3.11) is that this is a polynomial in $S_{i}^{a} \widetilde{S}_{b}^{j}$ of order $k$. (The fact that $S$ always appears together with $\widetilde{S}$ follows from symmetry.) SUSY vacua at finite distance in field space appear whenever $\partial_{S} \operatorname{det} \mathcal{M}=\partial_{\widetilde{S}} \operatorname{det} \mathcal{M}=0$. This is automatically satisfied for $S=\widetilde{S}=0$ and at some other isolated points at which $S, \widetilde{S} \sim m$. In all of these cases the meson VEV is of order $\langle Q \widetilde{Q}\rangle \sim m^{\frac{N_{f}}{N_{c}}-1} \Lambda^{3-\frac{N_{f}}{N_{c}}}$ and once written in terms 
of the magnetic variables it is clearly parametrically far away from the origin for $\mu \ll \Lambda$. Lastly, because $k \leq N_{f}-N_{c}$ there is a runaway behavior for large $S, \widetilde{S}$ (for example in the direction where they are all equal). Indeed, for large $S$ the superpotential (3.12) takes the

form $W_{e f f} \sim\left(m^{N_{f}-2 k} S^{2 k}\right)^{\frac{1}{N_{c}}} \Lambda^{3-\frac{N_{f}}{N_{c}}}$. (With a mass term for the singlets this runaway is stabilized.) On this branch some of the mesons are large and some are small.

Generally speaking, the fact that all the SUSY vacua and the runaway are far in field space is not surprising. This follows merely from the fact that these effects can be described by non-perturbative physics in the IR-free dual gauge theory. They must, therefore, not affect the structure of the theory at small VEVs.

\subsection{Orthogonal and Symplectic Groups}

First, we will consider $S O\left(N_{c}\right)$ gauge theory with $N_{f}$ flavors, $Q_{I}$ with $I=1 . . N_{f}$, in free magnetic range, namely $N_{c}-2<N_{f}<\frac{3}{2}\left(N_{c}-2\right)$. As in the $S U(N)$ case, we divide these electric quarks into two subsets, including $k$ and $N_{f}-k$ quarks labeled by the indices $i$ and $a$. Now we add singlets under the gauge group $S_{i a}$ and consider the following superpotential (with $m_{I J}$ being a full-rank symmetric matrix)

$$
W=m_{I J} \vec{Q}_{I} \cdot \vec{Q}_{J}+\lambda S_{i a} \vec{Q}_{i} \cdot \vec{Q}_{a}
$$

We have not included a mass term for $S$ although this is needed to make the theory generic. The reason is that, like in the case of $S U\left(N_{c}\right)$, including it does not affect the results of the analysis. The same comment holds for our $S p$ theory.

In the IR, the theory is described by its Seiberg dual with a magnetic group $S O(N \equiv$ $\left.N_{f}-N_{c}+4\right)$ 44]. The global symmetry of the model is $S O(k) \times S O\left(N_{f}-k\right)$. If the coupling $\lambda$ in (3.14) vanishes and the matrix $m_{I J}$ is proportional to the identity, the symmetry is enhanced to $S O\left(N_{f}\right)$. To further simplify the discussion we again take $k=N$ and in order to preserve a global symmetry $S O(N) \times S O\left(N_{c}-4\right)$ we choose the quark mass matrix to be $m_{I J}=m_{1} \delta_{i j} \oplus m_{2} \delta_{a b}$. In the IR, the superpotential is

$$
W=q^{T} M q-\mu_{1}^{2} M_{i i}-\mu_{2}^{2} M_{a a}+\lambda \Lambda S_{a i} M_{a i}
$$

Here the magnetic meson $M$ is a symmetric $N_{f} \times N_{f}$ matrix and unlike in the $S U(N)$ case there are, of course, no conjugate quarks. 
Assuming that $\lambda \sim 1$ and integrating out the heavy fields $S_{a i}$ and $M_{i a}$ we find an IR effective theory which is very similar to what we had in the case of $S U(N)$. The SUSY-breaking vacuum of the model is obtained for

$$
q^{T}=\left(\mu_{1} \mathbb{I}_{N \times N}, \mu_{2} \mathbb{I}_{N \times N}, 0\right), \quad M_{I J}=0 .
$$

The vacuum energy of this state is $\left(2 N_{c}-N_{f}-8\right) \mu_{2}^{4}$. At the scale $\mu_{2}$, the symmetry is broken as follows (as before, we decompose the magnetic quarks as $q=(\chi, \lambda)$ ):

$$
S O(N)_{\chi} \times\left[S O\left(N_{c}-4\right) \times S O(N)_{m a g}\right] \hookrightarrow S O(N)_{\chi} \times S O(N)_{\text {diagonal }} \times S O\left(2 N_{c}-N_{f}-8\right)
$$

At the scale $\mu_{1}$ this global symmetry is further broken down to

$$
S O(N)_{\chi} \times S O(N)_{\text {diagonal }} \times S O\left(2 N_{c}-N_{f}-8\right) \hookrightarrow S O(N)_{\text {visible }} \times S O\left(2 N_{c}-N_{f}-8\right)
$$

Analogously to the $S U(N)$ theory, there are some Nambu-Goldstone bosons and some pseudo-moduli (which are stabilized at one-loop).

The situation is also very similar in the $S p\left(N_{c}\right)$ theory with $2 N_{f}$ electric quarks in the fundamental representation. The free magnetic phase occurs for $\left(N_{c}+3\right) \leq N_{f}<$ $\frac{3}{2}\left(N_{c}+1\right) .9$ We again divide our quarks into two sets, which include $2 N$ and $2\left(N_{f}-N\right)$ quarks, where $N \equiv N_{f}-N_{c}-2$. Introducing new gauge singlets $S_{a i}$ we postulate the following superpotential

$$
W=-m_{I J} Q_{\alpha}^{I} Q_{\beta}^{J} J_{\alpha \beta}+\lambda S_{i a} Q_{\alpha}^{i} Q_{\beta}^{a} J_{\alpha \beta}
$$

The breaking occurs largely along the lines of $S O(N)$ and $S U(N)$ and will not repeat all the steps here. However, we note that the global symmetry of the model with the superpotential (3.19) is $S p(N) \times S p\left(N_{f}-N\right)$. In the minimum of the IR effective theory this group is spontaneously broken down to $S p(N)_{v i s i b l e} \times S p\left(N_{f}-2 N\right)$. This is triggered again by mixing with the magnetic gauge group, as in the previous two cases.

\footnotetext{
9 In this analysis we follow the conventions of [9].
} 


\section{Comments on Phenomenology}

This section deals with two closely related topics. We first take a closer look at the phenomenology of the dynamical models we constructed above. Specifically, we will discuss various possibilities for breaking the accidental $R$-symmetry and roughly estimate some soft masses.

In the second part of this section we attempt to obtain a broader view of the phenomenological possibilities. Recall that the investigation of models of messengers teaches us a lot about gauge mediation, and in the same spirit, the analysis of models such as those in section 2 should be a good guide to many dynamical models of gaugino mediation (including those we discuss in this paper). We will see that many novel phenomenological scenarios are naturally embedded in such theories.

\subsection{Accidental R-Symmetry Breaking and Soft Masses}

Consider the $S U(N)$ theory we described in subsection 3.1. For simplicity we choose $N=5$. We identify $S U(5)_{\chi}$ with the GUT group. Then we notice that $S U(5)_{\text {diagonal }}$ from (3.6) plays the role of $G_{2}^{S M}$ from section 2, and the fields $\chi, \tilde{\chi}$ are the link fields. The model we discuss has an accidental $R$-symmetry which is not broken in the meta-stable vacuum, and therefore, gaugino masses do not emerge. To render the model realistic this accidental symmetry should be broken (spontaneously or explicitly). Breaking this symmetry is a technical issue and there are many ways to do it (relevant references can be found in the review [10]). For instance, small quartic deformations in the UV become relevant in the IR and can easily lead to $R$-symmetry breaking, either spontaneous or explicit. 10

To be concrete let us focus on adding a quartic deformation in the UV, which in the IR becomes

$$
\delta W=\frac{\epsilon \mu_{2}}{2}\left(\operatorname{Tr} Z^{2}\right) .
$$

This deformation was studied in detail, for example, in [46, 47]. One finds that (4.1) shifts the VEV of $Z$ away from the origin to 1 .

$$
Z \sim \frac{\epsilon \mu_{2}}{\left(\alpha_{h} / 4 \pi\right)} \mathbb{I} .
$$

10 Various models of messengers with $R$-symmetry breaking effects have been studied in some generality in 45 .

11 One could also imagine a double-trace deformation $(\operatorname{Tr} Z)^{2}$, but this does not affect the discussion qualitatively, for a dedicated study the reader is referred to [47. 
In this formula $\alpha_{h}=h^{2} / 4 \pi$ with $h$ being the Yukawa coupling in the Seiberg dual. We have set this coupling to one throughout the paper but in this formula we reintroduce it in order to make the parametric dependence manifest. In practice, the constraints of metastability and the structure of the one loop effective potential do not allow us to probe arbitrary values of $Z$.

The main point here is that in spite of the fact that the deformation (4.1) breaks the $R$-symmetry explicitly, the spontaneous breaking through $\langle Z\rangle$ is larger by an inverse loop factor. Thus, effectively, we get spontaneous breaking. This point is of conceptual importance, because by the Nelson-Seiberg theorem [48], explicit breaking would be in tension with metastability [49,50]. Indeed, in realizations such as (4.2), new vacua emerge in the IR effective theory, but the transition rate between our vacuum and these new vacua is parametrically suppressed.

There are several other ideas which lead to very similar effective descriptions. For example, if massless electric quarks are introduced, we can use the mechanism of 51] to probe large values of $\langle Z\rangle$ as well. To list a few other options, there is the baryonic deformation of [52] or models like in [53,54].

Because the breaking is mostly spontaneous, we can understand the physics without going example by example. We study the theory as a function of the pseudo-modulus VEV $\langle Z\rangle$. The phenomenological predictions then depend on $\langle Z\rangle$ and can be translated immediately to microscopic realizations upon expressing the VEV in terms of the microscopic variables.

It is well know that gaugino masses as a function of $\langle Z\rangle$ are only formed at the subleading order in SUSY-breaking parameter. Our simple model does not have a strict small SUSY breaking expansion parameter. (It can be easily introduced by further allowing the electric quarks have different masses, but we will not carry out this analysis here.) The absence of a leading order contribution to the gaugino mass is curiously reflected in a numerical $\sim 0.1$ suppression. The rough estimate of the soft gaugino mass as a function of $\langle Z\rangle$ is then

$$
m_{g} \sim 0.1 \frac{\alpha}{4 \pi}\left\{\begin{array}{clrl}
\mu_{2}^{6} /\langle Z\rangle^{5} & , & \langle Z\rangle & \gg \mu_{2} \\
\mu_{2} & , & \langle Z\rangle & \sim \mu_{2} \\
\langle Z\rangle & , & \langle Z\rangle & \ll \mu_{2}
\end{array}\right.
$$

This rather small contribution to the gaugino soft mass posed severe phenomenological troubles in gauge-mediation model-building based on ISS. However, here it is less of a problem because the scalar masses can be screened as well. There are also models which produce 
gaugino masses at leading order, as in (2.1), including [35,36]. We will not elaborate on these models, but we would like to note that we believe many of their phenomenological features can be drastically improved in our framework.

We now switch to discussing the soft scalar masses. The two-loop contribution has the $v^{2} / M^{2}$ suppression factor we discussed at length in section 2 , and in our model the result can be estimated as

$$
m_{\widetilde{f}}^{2} \sim\left(\frac{\alpha}{4 \pi}\right)^{2} \mu_{1}^{2}
$$

This estimate of the two-loop contribution holds when $\mu_{1}^{2} \ll \mu_{2}^{2}$. It is independent of $\langle Z\rangle$ and of the ratio $\frac{\mu_{1}}{\mu_{2}}$ due to a peculiar see-saw like messenger structure for $\langle Z\rangle \gg \mu_{2} .12$ In more detail, since we have chosen to study the simplest version of the model, we find that, for all $\langle Z\rangle$, the messenger masses and mass splittings are comparable. Equation (4.4) can therefore be understood as a result of the usual screening formula $m_{\widetilde{f}}^{2} \sim\left(\frac{\alpha}{4 \pi}\right)^{2} \frac{\mu_{1}^{2}}{M^{2}} \frac{F^{2}}{M^{2}}$ with $F \sim M^{2}$. Of course, since $\mu_{1}$ is smaller than all the other parameters in the problem, (4.4) reflects a screened contribution to the scalar masses.

Very importantly, there are also three-loop contributions which could be even larger than (4.4), depending on the parameters. The idea is that the $\chi$ quarks have some soft masses at two-loops, roughly given by $\frac{\alpha^{2}}{16 \pi^{2}} \mu_{2}^{2}$. These $\chi$ quarks then play the role of the scalar component of a massive gauge multiplet, and therefore this feeds to the visible particles. This contribution is estimated as

$$
m_{\widetilde{f}}^{2} \sim \frac{\alpha^{3}}{(4 \pi)^{3}} \mu_{2}^{2}
$$

This estimate is applicable as long as $\mu_{1}^{2}>\frac{\alpha^{2}}{16 \pi^{2}} \mu_{2}^{2}$, which is definitely the regime one should be interested in anyway. A more careful analysis also shows that there is no logarithmic enhancement of (4.5) but we will not go into details here since this will take us too far afield (see [56] for a related discussion). We see that depending on the comparison of $\mu_{1}^{2} / \mu_{2}^{2}$ to a loop factor, this can be negligible or dominant compared to (4.4). More interestingly, if the gaugino mass does not arise at leading order, we expect (4.5) to be more important than the usual gaugino mediated contribution (2.5).

Another parenthetical issue about this specific model is that some of the $\lambda, \widetilde{\lambda}$ scalars are massless. Since those particles act as messengers and are charged under the SM this feature

12 This peculiarity, in fact, appears in many O'R-like models. It was systematically studied in 55. 
should be removed. This particular problem is trivially fixed, often by the same source that breaks $R$-symmetry. In addition, these massless scalars automatically disappear once we gauge flavor symmetries. Lastly, these massless particles disappear if we allow a more general mass matrix in the UV Lagrangian. (This is also a useful way to construct theories which are not necessarily at some relatively low scale.)

Finally, we would like to note that dynamical models with large flavor symmetries and which are naturally perturbative up to the GUT scale are not known, and our model does not change this state of affairs. However, theories which reduce to the deconstructed ansatz are expected to have a smaller beta function than usual. Indeed, models based on ISS like those we tried to encompass in the analysis above, when fitted into our framework, generally behave better than their original incarnations. 13

\subsection{Spectroscopy}

Even the simple theory we discussed above, as a function of its parameters and the choice of how to break the $R$-symmetry, can give rise to a diverse spectrum. It varies from a theory with an unnatural spectrum (the sparticles being significantly heavier than the gauginos) all the way to some form of gaugino mediation. In our particular scenario we chose to focus on a theory which is naturally mediating SUSY breaking at a low scale, but as we remarked, the mediation scale can trivially be promoted to a free parameter. Therefore, we should consider both high and low scale mediation scenarios.

In order to get some handle over these various phenomenological scenarios, it is convenient to step back and reconsider the ansatz of section 2. In particular, we can define its simplest version as "minimal gaugino mediation." It consists of a sector of link fields which are forced to get VEVs due to a Lagrange multiplier $\mathcal{A}$ in the superpotential $\int d^{2} \theta \mathcal{A}\left(L \widetilde{L}-v^{2}\right)$. The Hidden sector $H$ consists of a SUSY-breaking spurion $X=M+\theta^{2} F$ which couples to a single pair of messengers $X \psi \widetilde{\psi}$.

We see that this minimal model has three dimensionful scales, one more than minimal gauge mediation. As a function of $v / M$ for large $v$ (say a little below the GUT scale) this model interpolates between high scale minimal gauge mediation (see the review [57]) and

13 In deconstructed models the gauge couplings are shifted by the Higgs mechanism. Unless this is done in an $S U(5)$ invariant way (or some other special relations are satisfied) perturbative unification is ruined. This is similar to constraints on the messenger fields in ordinary models of gauge mediation. 
high scale gaugino mediation (considered, for example, in [58). For low $v$ (e.g. 10 TeV) the model interpolates between low scale minimal gauge mediation and low scale gaugino mediation. The phenomenology of the latter scenario is much less studied, but the analysis of [56,59] shows convincingly that such theories, even in their minimal form, lead to spectacular collider signatures.

To the best of our knowledge, the particle spectrum has not been systematically computed as a function of $v / M$. Given that it interpolates between such canonical (and compelling) scenarios, it cannot be overemphasized how important it is to understand the intermediate regime.

We see that even for "minimal gaugino mediation" the phenomenology (and particle spectrum as a function of the parameters) is largely unknown, it is then not surprising that the simplest and most interesting generalizations of it have not been studied at all. Even though these models fall into the class of GGM, a lot of the "common lore" about gauge mediation can be violated. As a first example, some of the general results in 60] about theories of messengers do not hold in our theories; the bino can be pretty heavy. Further, we can take into account doublet-triplet splitting in the messenger sector or in the link field spectra.14 We expect that such models give rise to exotic spectra whose signatures have not been studied. For instance, it is conceivable that the usual hierarchy between the sparticles $m_{\tilde{q}}>m_{\widetilde{L}}>m_{\widetilde{e}_{R}}$ can be altered. This phenomenon, which is not standard regardless of the masses of the gauginos, can lead to surprising consequences if the gauginos are relatively heavy. For example, we might find a sneutrino NLSP. Even if the scalars are not all lighter than the gauginos, a reversed ordering of the scalars in the spectrum might be very interesting as well.

Models like those of section 2 may have looked unnatural before, but we believe that the fact that they arise from perfectly natural (and generic) dynamical mechanisms, makes them very well motivated phenomenologically and warrants a careful investigation of their possible phenomenological signatures.

14 Analogous studies in gauge-mediation scenarios led to novel and surprising collider signatures, see e.g. 61-64. 


\section{Summary and Open Questions}

In this note, we showed that gaugino mediation can arise dynamically in asymptotically free gauge theories. Using Seiberg duality, we were able to find calculable examples where the screening of soft masses arises through higgsing of the magnetic gauge group. We expect calculable examples to be abundant with low energy effective descriptions given by various gauged WZ-models resembling Fig.1.

The scalar screening necessary for gaugino mediation has commonly been considered an extra dimensional or strongly coupled phenomena. While these examples do arise in the strongly coupled regime of the "electric" description, they are fully calculable. Because these models dynamically give rise to a deconstruction-like Lagrangian, it suggests that other "extra-dimensional phenomena" may also emerge from simple single sector four dimensional theories. For example, it would be natural to study models of flavor by modifying the models presented here.

From a more phenomenological perspective, the spectra of particles in these models obey the rules of general gauge mediation. However, given a generic model, the resulting spectrum would appear to be quite unconventional. Given how naturally these models arise within SUSY gauge theories, these models warrant further phenomenological study.

We close by listing few of the many open questions which we believe are worth pursuing. As far as dynamical models are concerned, we have only discussed the tip of the iceberg of possibilities. It should be better understood which theories are capable of screening the scalar masses and what common features they share. Our phenomenological knowledge of such theories is very limited unfortunately; even the spectrum of soft masses in the minimal effective model is not know as a function of its basic parameters. There are excellent reasons to believe that these theories lead to interesting spectra and novel collider signatures. Therefore, it would be nice to systematically study such models. As a starting point for such a systematic study, it would be useful to understand better all the different kinds of contributions to the visible soft masses. Finally, it is also tempting to try and embed a mechanism for the $\mu / B_{\mu}$ terms in these theories. 


\section{Acknowledgments:}

We would like to thank R. Argurio, N. Craig, T. Dumitrescu, A. Giveon, N. Seiberg, D. Shih, and M. Sudano for comments. The work of DG was supported in part by DOE grant DE-FG02-90ER40542. AK was partially supported by NSF grant PHY-0801323. The work of ZK was supported in part by NSF grant PHY-0503584. Any opinions, findings, and conclusions or recommendations expressed in this material are those of the authors and do not necessarily reflect the views of the National Science Foundation. 


\section{References}

[1] M. Dine and W. Fischler, "A Phenomenological Model Of Particle Physics Based On Supersymmetry," Phys. Lett. B 110, 227 (1982).

[2] S. Dimopoulos and S. Raby, "Supercolor," Nucl. Phys. B 192, 353 (1981).

[3] C. R. Nappi and B. A. Ovrut, "Supersymmetric Extension Of The SU(3) X SU(2) X U(1) Model," Phys. Lett. B 113, 175 (1982).

[4] M. Dine, A. E. Nelson, Y. Nir and Y. Shirman, "New tools for low-energy dynamical supersymmetry breaking," Phys. Rev. D 53, 2658 (1996) arXiv:hep-ph/9507378.

[5] L. Alvarez-Gaume, M. Claudson and M. B. Wise, "Low-Energy Supersymmetry," Nucl. Phys. B 207, 96 (1982).

[6] M. Dine and W. Fischler, "A Supersymmetric Gut," Nucl. Phys. B 204, 346 (1982).

[7] M. Dine, A. E. Nelson and Y. Shirman, "Low-Energy Dynamical Supersymmetry Breaking Simplified," Phys. Rev. D 51, 1362 (1995) arXiv:hep-ph/9408384].

[8] M. Dine and A. E. Nelson, "Dynamical supersymmetry breaking at low-energies," Phys. Rev. D 48, 1277 (1993) arXiv:hep-ph/9303230.

[9] K. A. Intriligator, N. Seiberg and D. Shih, "Dynamical SUSY breaking in meta-stable vacua," JHEP 0604, 021 (2006) arXiv:hep-th/0602239.

[10] R. Kitano, H. Ooguri and Y. Ookouchi, "Supersymmetry Breaking and Gauge Mediation," arXiv:1001.4535 [hep-th].

[11] Z. Komargodski and D. Shih, "Notes on SUSY and R-Symmetry Breaking in WessZumino Models," JHEP 0904, 093 (2009) [arXiv:0902.0030 [hep-th]].

[12] S. Shirai, M. Yamazaki and K. Yonekura, "Aspects of Non-minimal Gauge Mediation," JHEP 1006, 056 (2010) [arXiv:1003.3155 [hep-ph]].

[13] D. E. Kaplan, G. D. Kribs and M. Schmaltz, "Supersymmetry breaking through transparent extra dimensions," Phys. Rev. D 62, 035010 (2000) arXiv:hep-ph/9911293.

[14] Z. Chacko, M. A. Luty, A. E. Nelson and E. Ponton, "Gaugino mediated supersymmetry breaking," JHEP 0001, 003 (2000) arXiv:hep-ph/9911323.

[15] J. R. Ellis, K. Enqvist and D. V. Nanopoulos, "A Very Light Gravitino In A No Scale Model," Phys. Lett. B 147, 99 (1984).

[16] J. R. Ellis, C. Kounnas and D. V. Nanopoulos, "No Scale Supersymmetric Guts," Nucl. Phys. B 247, 373 (1984).

[17] N. Arkani-Hamed, A. G. Cohen and H. Georgi, "(De)constructing dimensions," Phys. Rev. Lett. 86, 4757 (2001) [arXiv:hep-th/0104005].

[18] C. T. Hill, S. Pokorski and J. Wang, "Gauge invariant effective Lagrangian for KaluzaKlein modes," Phys. Rev. D 64, 105005 (2001) arXiv:hep-th/0104035.

[19] H. C. Cheng, D. E. Kaplan, M. Schmaltz and W. Skiba, "Deconstructing gaugino mediation," Phys. Lett. B 515, 395 (2001) [arXiv:hep-ph/0106098]. 
[20] C. Csaki, J. Erlich, C. Grojean and G. D. Kribs, "4D constructions of supersymmetric extra dimensions and gaugino mediation," Phys. Rev. D 65, 015003 (2002) arXiv:hepph/0106044.

[21] T. S. Roy and M. Schmaltz, "A hidden solution to the $\mu / B_{\mu}$ problem in gauge mediation," Phys. Rev. D 77, 095008 (2008) [arXiv:0708.3593 [hep-ph]].

[22] H. Murayama, Y. Nomura and D. Poland, "More Visible Effects of the Hidden Sector," Phys. Rev. D 77, 015005 (2008) arXiv:0709.0775 [hep-ph]].

[23] N. Seiberg, "Electric - magnetic duality in supersymmetric nonAbelian gauge theories," Nucl. Phys. B 435, 129 (1995) arXiv:hep-th/9411149.

[24] P. Meade, N. Seiberg and D. Shih, "General Gauge Mediation," Prog. Theor. Phys. Suppl. 177, 143 (2009) [arXiv:0801.3278 [hep-ph]].

[25] L. M. Carpenter, M. Dine, G. Festuccia and J. D. Mason, "Implementing General Gauge Mediation," Phys. Rev. D 79, 035002 (2009) arXiv:0805.2944 [hep-ph]].

[26] K. Benakli and M. D. Goodsell, "Dirac Gauginos in General Gauge Mediation," Nucl. Phys. B 816, 185 (2009) [arXiv:0811.4409 [hep-ph]].

[27] L. M. Carpenter, "Surveying the Phenomenology of General Gauge Mediation," arXiv:0812.2051 [hep-ph].

[28] M. Buican, P. Meade, N. Seiberg and D. Shih, "Exploring General Gauge Mediation," JHEP 0903, 016 (2009) [arXiv:0812.3668 [hep-ph]].

[29] D. Marques, "Generalized messenger sector for gauge mediation of supersymmetry breaking and the soft spectrum," JHEP 0903, 038 (2009) [arXiv:0901.1326 [hep-ph]].

[30] M. Luo and S. Zheng, "Gauge Extensions of Supersymmetric Models and Hidden Valleys," JHEP 0904, 122 (2009) [arXiv:0901.2613 [hep-ph]].

[31] A. Rajaraman, Y. Shirman, J. Smidt and F. Yu, "Parameter Space of General Gauge Mediation," Phys. Lett. B 678, 367 (2009) arXiv:0903.0668 [hep-ph]].

[32] S. Abel, M. J. Dolan, J. Jaeckel and V. V. Khoze, "Phenomenology of Pure General Gauge Mediation," JHEP 0912, 001 (2009) arXiv:0910.2674 [hep-ph]].

[33] T. Kobayashi, Y. Nakai and R. Takahashi, "Fine Tuning in General Gauge Mediation," JHEP 1001, 003 (2010) [arXiv:0910.3477 [hep-ph]].

[34] M. McGarrie and R. Russo, "General Gauge Mediation in 5D," Phys. Rev. D 82, 035001 (2010) arXiv:1004.3305 [hep-ph]].

[35] R. Kitano, H. Ooguri and Y. Ookouchi, "Direct mediation of meta-stable supersymmetry breaking," Phys. Rev. D 75, 045022 (2007) [arXiv:hep-ph/0612139].

[36] A. Giveon, A. Katz and Z. Komargodski, "Uplifted Metastable Vacua and Gauge Mediation in SQCD," JHEP 0907, 099 (2009) [arXiv:0905.3387 [hep-th]].

[37] R. Auzzi, S. Elitzur and A. Giveon, "On Uplifted SUSY-Breaking Vacua and Direct Mediation in Generalized SQCD," JHEP 1003, 094 (2010) [arXiv:1001.1234 [hep-th]].

[38] N. Maru, "Direct Gauge Mediation of Uplifted Metastable Supersymmetry Breaking in Supergravity," arXiv:1008.1440 [hep-ph]. 
[39] E. Barnes, K. A. Intriligator, B. Wecht and J. Wright, "Evidence for the strongest version of the $4 \mathrm{~d}$ a-theorem, via a-maximization along RG flows," Nucl. Phys. B 702, 131 (2004) arXiv:hep-th/0408156.

[40] S. Franco and S. Kachru, "Single-Sector Supersymmetry Breaking in Supersymmetric QCD," Phys. Rev. D 81, 095020 (2010) arXiv:0907.2689 [hep-th]].

[41] N. Craig, R. Essig, S. Franco, S. Kachru and G. Torroba, "Dynamical Supersymmetry Breaking, with Flavor," Phys. Rev. D 81, 075015 (2010) [arXiv:0911.2467 [hep-ph]].

[42] A. Amariti, L. Girardello, A. Mariotti and M. Siani, "Metastable Vacua in Superconformal SQCD-like Theories," arXiv:1003.0523 [hep-th].

[43] S. Schafer-Nameki, C. Tamarit and G. Torroba, "A Hybrid Higgs," arXiv:1005.0841 [hep-ph].

[44] K. A. Intriligator and N. Seiberg, "Duality, monopoles, dyons, confinement and oblique confinement in supersymmetric $\mathrm{SO}(\mathrm{N}(\mathrm{c})$ ) gauge theories," Nucl. Phys. B 444, 125 (1995) arXiv:hep-th/9503179.

[45] D. Marques and F. A. Schaposnik, "Explicit R-Symmetry Breaking and Metastable Vacua," JHEP 0811, 077 (2008) [arXiv:0809.4618 [hep-th]].

[46] A. Giveon and D. Kutasov, "Stable and Metastable Vacua in SQCD," Nucl. Phys. B 796, 25 (2008) arXiv:0710.0894 [hep-th]].

[47] R. Essig, J. F. Fortin, K. Sinha, G. Torroba and M. J. Strassler, "Metastable supersymmetry breaking and multitrace deformations of SQCD," JHEP 0903, 043 (2009) arXiv:0812.3213 [hep-th]].

[48] A. E. Nelson and N. Seiberg, "R symmetry breaking versus supersymmetry breaking," Nucl. Phys. B 416, 46 (1994) arXiv:hep-ph/9309299.

[49] K. A. Intriligator and N. Seiberg, "Lectures on Supersymmetry Breaking," Class. Quant. Grav. 24, S741 (2007) arXiv:hep-ph/0702069].

[50] K. A. Intriligator, N. Seiberg and D. Shih, "Supersymmetry Breaking, R-Symmetry Breaking and Metastable Vacua," JHEP 0707, 017 (2007) arXiv:hep-th/0703281.

[51] A. Giveon, A. Katz, Z. Komargodski and D. Shih, "Dynamical SUSY and R-symmetry breaking in SQCD with massive and massless flavors," JHEP 0810, 092 (2008) arXiv:0808.2901 [hep-th]].

[52] S. Abel, C. Durnford, J. Jaeckel and V. V. Khoze, "Dynamical breaking of $U(1)_{R}$ and supersymmetry in a metastable vacuum," Phys. Lett. B 661, 201 (2008) arXiv:0707.2958 [hep-ph]].

[53] M. Dine and J. Mason, "Gauge mediation in metastable vacua," Phys. Rev. D 77, 016005 (2008) arXiv:hep-ph/0611312.

[54] N. Haba and N. Maru, "A Simple Model of Direct Gauge Mediation of Metastable Supersymmetry Breaking," Phys. Rev. D 76, 115019 (2007) arXiv:0709.2945 [hep$\mathrm{ph}]]$. 
[55] A. Katz, "On the Thermal History of Calculable Gauge Mediation," JHEP 0910, 054 (2009) [arXiv:0907.3930 [hep-th]].

[56] A. De Simone, J. Fan, M. Schmaltz and W. Skiba, "Low-scale gaugino mediation, lots of leptons at the LHC," Phys. Rev. D 78, 095010 (2008) [arXiv:0808.2052 [hep-ph]].

[57] G. F. Giudice and R. Rattazzi, "Theories with gauge-mediated supersymmetry breaking," Phys. Rept. 322, 419 (1999) arXiv:hep-ph/9801271].

[58] D. E. Kaplan and T. M. P. Tait, "Supersymmetry breaking, fermion masses and a small extra dimension," JHEP 0006, 020 (2000) arXiv:hep-ph/0004200].

[59] A. De Simone, J. Fan, V. Sanz and W. Skiba, "Leptogenic Supersymmetry," Phys. Rev. D 80, 035010 (2009) arXiv:0903.5305 [hep-ph]].

[60] T. T. Dumitrescu, Z. Komargodski, N. Seiberg and D. Shih, "General Messenger Gauge Mediation," JHEP 1005, 096 (2010) [arXiv:1003.2661 [hep-ph]].

[61] C. Cheung, A. L. Fitzpatrick and D. Shih, "(Extra)Ordinary Gauge Mediation," JHEP 0807, 054 (2008) arXiv:0710.3585 [hep-ph]].

[62] P. Meade, M. Reece and D. Shih, "Prompt Decays of General Neutralino NLSPs at the Tevatron," JHEP 1005, 105 (2010) [arXiv:0911.4130 [hep-ph]].

[63] A. Katz and B. Tweedie, "Signals of a Sneutrino (N)LSP at the LHC," Phys. Rev. D 81, 035012 (2010) [arXiv:0911.4132 [hep-ph]].

[64] A. Katz and B. Tweedie, "Leptophilic Signals of a Sneutrino (N)LSP and Flavor Biases from Flavor-Blind SUSY," Phys. Rev. D 81, 115003 (2010) arXiv:1003.5664 [hep-ph]]. 